\title{
Análisis de la ocupación humana de la cueva de Praileaitz I (Deba, Gipuzkoa): Paleolítico Superior y Epipaleolítico
}

\author{
Praileaitz I (Deba, Gipuzkoa): Praileaitz I haitzuloaren giza-okupazioaren analisia: \\ Goi Paleolitoa eta Epipaleolitoa \\ Study of the human occupation in Praileaitz I cave (Deba, Gipuzkoa) in the \\ Upper Palaeolithic and the Epipalaeolithic
}

PALABRAS CLAVE: Praileaitz I, Solutrense, Magdaleniense Inferior, Magdaleniense Superior-Final, Epipaleolítico, Contexto arqueológico. GAKO-HITZAK: Praileaitz I, Solutre Aldia Behe Madeleine Aldia, Goi-Azken Madeleine, Epipaleolitoa, Testuinguru arqueológikoa. KEYWORDS: Praileaitz I, Solutrean, Lower Magdalenian, Upper-End Magdalenian, Epipaleolithic, Archaelological Context.

Xabier PEÑALVER ${ }^{(1)}$, Sonia SAN JOSE(2), Jose Antonio MUJIKA-ALUSTIZA ${ }^{(3)}$

\section{RESUMEN}

Se presenta una síntesis de los resultados obtenidos en la intervención arqueológica de la cueva de Praileaitz I entre el año 2000 y el 2009 y de los diferentes estudios realizados, agrupándolos en función de los períodos presentes. Así mismo, se hace referencia al contexto arqueológico de la zona en los momentos de ocupación de la cueva intervenida.

\section{LABURPENA}

Artikulu honen gaia Praileaitz I haitzuloan 2000tik 2009ra egindako esku-hartze arkeologikoaren emaitzen eta egindako ikerketen sintesia da, aroaren arabera multzokatuta. Era berean, aintzat hartu dugu haitzuloaren okupazio uneetan zegoen testuinguru arkeologikoa.

\section{ABSTRACT}

This essay presents a synthesis of the human occupation of the cave Praileaitz I, based on the results of the archaeological excavations between years 2000 and 2009 and on the studies carried out, classifying them according to the existing periods. It also makes reference to the archaeological context of the area in the periods that the studied cave was occupied.

\section{1.- PRAILEAITZ I. ENTORNO GEOGRÁFICO Y ARQUEOLÓGICO}

La cueva de Praileaitz I se abre en las masas calizas del macizo de Izarraitz sobre los meandros existentes en el último tramo del río Deba, dentro del barrio de Sasiola (PEÑALVER, 2014; PEÑALVER et al., 2006; SAN JOSE et al., 2017). Se trata de una zona de relieves con formas más o menos cónicas, relativamente aislados entre sí, y con altitudes de en torno a $130 \mathrm{~m}$ sobre el nivel del mar (ARANBURU et al. , 2017).

En su entorno más próximo se han realizado investigaciones arqueológicas en diversas cuevas, cuya importancia varía notablemente en función de su estratigrafía y riqueza material. Indudablemente destaca la cueva de Ermittia, a unos $350 \mathrm{~m}$ de la de Praileaitz I, dentro de un complejo paisaje kárstico. Su yacimiento, que con- servaba una notable e importante estratigrafía centrada entre el Solutrense y la época romana, aunque también con episodios de abandono o livianas ocupaciones, ha sido profundamente alterado en distintos momentos. Así mismo, muy próxima a Praileaitz I se encontraba Praileaitz II, excavada por E. Uribarri (1988 y 1989), con una estratigrafía menos compleja y ocupaciones del Paleolítico Superior y del Holoceno, además de alguna evidencia postpaleolítica y romana.

Otros yacimientos excavados en el mismo entorno y situados en la otra orilla del Deba, como Langatxo e Iruroin, presentan niveles que se reducen al Magdaleniense Final, además de alguna reutilización postpaleolítica (ZUMALABE, 1994 y 1999).

Por otra parte, cabe señalar la presencia de otras tres cavidades algo más alejadas, dos de las cuales pu-

\footnotetext{
(1) Departamento de Arqueología Prehistórica. Sociedad de Ciencias Aranzadi. Zorroagaina, z.g. 20014 Donostia. praileaitz@gmx.com

(2) Departamento de Arqueología Prehistórica. Sociedad de Ciencias Aranzadi.

(3) Facultad de Letras (Universidad del País Vasco -UPV-EHU-). Tomás y Valiente s/n. Vitoria-Gasteiz.
} 
dieron haber estado en estrecha relación con Praileaitz I durante el Magdaleniense Superior-Final y el Aziliense, como son Aizkoltxo (Mendaro) -situada a unos cuatro kilómetros curso arriba del río Deba- (EDESO, MUJIKAALUSTIZA, 2006) o Urtiaga (Deba) -ubicada a unos 4 $\mathrm{km}$ hacia el este-; esta última cuenta con una larga secuencia estratigráfica, perfectamente paralelizable con la de Ermittia, abarcando desde el Solutrense a la Edad del Bronce (MUJIKA, 1993; ESPARZA, MUJIKA, 1999). La tercera de estas cavidades, Santa Catalina (Lekeitio), se abre a una quincena de kilómetros, y presenta una interesante estratigrafía con hallazgos singulares de finales del Pleistoceno e inicios del Holoceno (BERGANZA et al., 2014).

Volviendo a Praileaitz I, esta cavidad se sitúa a 3 $\mathrm{km}$ de la costa, a unos $65 \mathrm{~m}$ sobre el cauce del río, en una zona en la que en la actualidad se aprecian los efectos de las mareas. Sin embargo, esto no siempre ha sido así, ya que, como señala J. M. Edeso en su estudio (2017), la línea de costa ha conocido distintos avances y retrocesos, siendo Praileaitz I una cavidad relativamente alejada de la costa a lo largo del Tardiglaciar, llegando a ser esa distancia de casi una docena de kilómetros. A lo largo del Holoceno, en cambio, el mar avanzará progresivamente hasta llegar a aproximarse a un kilómetro de la cueva. Por otra parte, según las curvas batimétricas, las zonas actualmente sumergidas tendrían características muy diferentes a las del propio entorno de la cavidad, al ser el relieve suave, con unos paisajes y una fauna diferentes. En esta acogedora banda con orientación este-oeste, de fácil tránsito para las poblaciones paleolíticas, se ubicarían los asentamientos más estables, y principalmente los correspondientes a la estación más rigurosa del año. La movilidad de estos grupos les llevaría a acceder directamente a las distintas fuentes de sílex o a contactar con grupos más alejados que se abastecían directamente en los afloramientos, caso de los sílex de Salies de Béarn, Chalosse, o las distintas variedades del sílex de flysch.

Al mismo tiempo, los cursos de los distintos ríos que se internan en el territorio permitirían acercarse también a otros recursos abióticos (principalmente sílex de Urbasa y Treviño) y bióticos. Desgraciadamente, estos últimos son en la actualidad prácticamente desconocidos, y únicamente la simulación de distintos modelos del desarrollo de la vegetación potencial en el Pirineo Occidental y territorios limítrofes a lo largo de las distintas estaciones del año y en las condiciones climáticas correspondientes a cada periodo, así como las de la fauna potencial disponible atendiendo a su movilidad estacional, podrían permitirnos una aproximación a la gestión de los recursos practicada por estos grupos humanos.

En lo que se refiere al abastecimiento de sílex de Praileaitz I, a pesar de identificarse seis tipos de sílex diferentes, el del Flysch es el mayoritario con un $80,5 \%$, siguiendo en importancia el de Treviño (3,7\%), el de Urbasa $(2,6 \%)$ y el de Chalosse $(2,1 \%)$ (TARRIÑO, 2017).

\section{2.- LA CAVIDAD Y SU OCUPACIÓN}

La cueva de Praileaitz I está dividida en varios espacios bastante bien delimitados: un rellano exterior, el vestíbulo, la galería noroeste, el tránsito entre el vestíbulo y la primera sala, la primera y la segunda sala interior, además de otras zonas más alejadas de la boca a las que se accede por una serie de galerías, y en las que se han localizado pinturas consistentes en puntos y trazos rojos, la mayor parte de ellas sobre banderas (GARCÍADíEZ, OCHOA, 2017). La excavación se ha centrado en todos los espacios a excepción de esas zonas profundas, excavándose prácticamente de forma íntegra, y habiéndose abierto $191 \mathrm{~m}^{2}$ en los que se han puesto de manifiesto diversas ocupaciones paleolíticas y epipaleolíticas de intensidad y duración variables (PEÑALVER et al., 2017). De todas estas áreas será el vestíbulo el lugar en el que se ha detectado la estratigrafía más completa, probablemente por ser el espacio más acogedor, amplio y luminoso.

La presencia humana durante todos los episodios registrados en la cavidad es muy liviana, tanto que algunas de las fugaces ocupaciones son difíciles de contextualizar por el lento proceso de sedimentación que se produce. Esta dificultad aumenta a causa de las diferentes características del depósito en cada uno de los espacios, donde además de procesos de deposición, se producen otros de arrastre (ARANBURU et al., 2017; GARCIA-IBAIBARRIAGA et al., 2017) que explicarían la presencia de objetos de características tipológicas discordantes con el contexto en que fueron hallados. Los periodos de ocupación fueron breves como se deduce del bajo número de efectivos industriales (MUJIKA-ALUSTIZA, 2017), de la escasa fauna manipulada por sus pobladores (CASTAÑOS, CASTAÑOS, 2017) y de la poca diversidad de maderas utilizadas como combustible (RUIZ-ALONSO, ZAPATA, 2017).

Durante los periodos de desocupación, la cavidad fue guarida de carnívoros -oso (Ursus spelaeus/arctos), pantera (Panthera pardus), lobo (Canis lupus), etc.(CASTAÑOS, CASTAÑOS, 2017) y lugar de cobijo para aves - chova piquigualda (Pyrrhocorax graculus), chova piquirroja (Pyrrhocorax pyrrhocorax), cuervo (Corvus corax), urraca (Pica pica), etc.- (MORENO-GARCÍA, 2017). Hay que señalar que parte de los restos faunísticos corresponden a periodos anteriores a los de las industrias más antiguas recuperadas en su posición primaria, como es el caso de una muestra de Ursus spelaeus datada en 50100 BP y otra de Panthera pardus en $35010 \pm 280$ BP (40225-38853 cal BP ${ }^{1}$ ).

\subsection{Primeros asentamientos}

La datación absoluta más antigua en relación aparente con industria, obtenida en la zona de contacto del

\footnotetext{
${ }^{1}$ Dataciones calibradas usando la curva de calibración Intcal 13 (programa de calibración Oxcal 4.2).
} 
vestíbulo con la galería noroeste de Praileaitz I, es de 25320 140 BP (29774-28976 cal BP), y correspondería al Gravetiense. De todos modos, el contexto no acaba de ser del todo claro, ya que, entre los escasos restos hallados, no hay ningún objeto significativo desde el punto de vista cronocultural, aunque por el contrario se recogieron varios de carácter lítico (bifaz, raedera, etc.) que, por sus características tecnológicas y tipológicas, tendrían perfecta cabida en el Paleolítico Medio. Estas evidencias planteaban la cuestión de si habrían sido acarreadas durante algún episodio húmedo a través de la galería noroeste, por la cual se accedería a otra perpendicular a la misma, en donde recientemente se ha confirmado la existencia de restos de similar cronología (PEÑALVER, 2013).

La cavidad durante el Paleolítico Medio pudo, asimismo, haber sido utilizada como lugar de hibernación de osos (50100 B.P.), siendo así mismo coherente con la presencia de hiena y pantera (CASTAÑOS, CASTAÑOS, 2017).

Ya en los inicios del Paleolítico Superior antiguo (Chatelperroniense) se detectan restos de fauna en el abrigo exterior, punto elegido probablemente para recalar unas horas con las presas; la frecuencia de grandes carnívoros (oso de las cavernas (Ursus spelaeus), hiena (Crocuta crocuta) y leopardo (Panthera pardus)) justifican la presencia de restos de grandes bóvidos. A partir del Gravetiense se detecta la ocupación humana de las diferentes zonas del interior de la cavidad y desaparecen el oso de las cavernas y la hiena, siendo el primero sustituido por el oso pardo (CASTAÑOS, CASTAÑOS, 2017).

\subsection{El Solutrense}

Las primeras evidencias bien contextualizadas de presencia humana en Praileaitz I corresponden al Solutrense. Son muy pocos los restos industriales líticos y óseos atribuibles a esta fase, ya que únicamente se ha excavado el techo de este estrato, pero no hay dudas sobre su cronología debido a la existencia de varias piezas líticas de retoques planos (entre ellas un fragmento de punta, un buril sobre truncadura -en Antoliña existen también varios buriles laterales, AGUIRRE et al., 2000-), cuya tipología es clara. Esta ocupación se ve refrendada por una fecha obtenida en el vestíbulo (17760 $\pm 70 \mathrm{BP}$ -21785-21244 cal BP-) y otra de 17530 \pm 70 BP (2144020919 cal BP) correspondiente a la datación directa del fragmento de bastón perforado liso hallado en el inicio de la segunda sala. En este mismo contexto se recogió una azagaya decorada con toscos trazos transversales cuyos paralelos más próximos, aunque sin referencia estratigráfica, se hallaron en Aitzbitarte IV durante la excavación practicada a finales del siglo XIX. Ya en estratigrafía, durante la investigación de J. M. de Barandiaran en esta misma cueva, se halló un bastón perforado en asta de reno de cronología solutrense (MÚGICA, 1983: Fig.17).

Probablemente, los ocupantes solutrenses de Praileaitz I fueron coetáneos con los de Urtiaga, (cueva da- tada en $17050 \pm 140 \mathrm{BP}, 17170 \pm 350 \mathrm{BP}$ y $17730 \pm 290$ $\mathrm{BP})$, donde se registra un predominio de la caza de ciervo, seguida de la cabra, y los de Ermittia (datada en $16890 \pm 75$ BP y $17725 \pm 165$ BP), donde domina la caza de cabra, seguida del sarrio y el ciervo. Las ocupaciones de estas dos últimas cuevas eran de mayor entidad que la de Praileaitz I (ESPARZA, MUJIKA, 1999). En ésta, durante este período, la cabra es el animal más capturado, seguida del sarrio y del ciervo. Así, en el vestíbulo de la cueva, el sector más rico en fauna de todo el yacimiento, los restos de cabra ascienden a 577, siendo 200 y 143 los de sarrio y ciervo respectivamente. Especies como el caballo, el bóvido o el reno, están representadas con muy escasos restos (CASTAÑOS, CASTAÑOS, 2017). Durante este período, la presencia del Lagópodo Escandinavo, entre las aves, es una clara evidencia de unas condiciones ambientales frías (MORENO-GARCIA, 2017), tal como lo avala también la presencia de ciertos micromamíferos en esta época y en el Magdaleniense Inferior (GARCIA-IBAIBARRIAGA et al., 2017). En este sentido, la única información palinológica que se ha podido recuperar en la cueva permite hablar en este período de "un paisaje abierto con unos pocos pinos, enebros y abedules" (IRIARTE, 2017).

Dentro del contexto regional, en este período habría que subrayar la importancia de los yacimientos de Bolinkoba (Abadiño) y Atxuri (Mañaria), pero sobre todo el de Antoliña (Gautegiz-Arteaga), que cuenta con una nutrida colección de puntas de base cóncava (40 ejemplares), además de otro tipo de útiles, y que ha aportado las siguientes fechas: $19280 \pm 120 \mathrm{BP}(\mathrm{GrN}-23785)$ en el nivel Lmc, y 19020 \pm 120 BP (Beta-230284) en H3-Lmc; y 17340 \pm 100 BP (Beta-251301) para el superior Lmb (AGUIRRE et al. 2011). También existen evidencias (5 puntas con retoques planos bifaciales, una elevada proporción de dorsos, etc.) del final del Solutrense en el nivel Il de la cueva de Arlanpe (Lemoa), la cual ha sido datada en 17260 \pm 70 BP (Beta-261388) y 17160 \pm 70 BP (Beta261389) (RíOS, J. et al.: 2013).

Por otra parte, al este del río Deba, dentro de la cuenca del Urola, se localiza la cueva de Amalda (Zestoa),

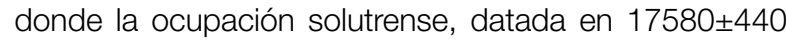
$\mathrm{BP}$, es evidente, contabilizándose 8 piezas de retoque plano (entre ellas 4-5 puntas de muesca y una de laurel), numerosos elementos de dorso, buriles, etc. (ALTUNA et al., 1990). Sería deseable la revisión de dicha colección, así como proceder a su datación sistemática, ya que su estratigrafía pudiera ser más compleja que la expuesta en la memoria, con probable presencia de alguna ocupación del Magdaleniense Inferior por la presencia de algún geométrico -triángulo y segmento- (CORCHÓN, 1993); esta hipótesis parece confirmarse por dos fechas

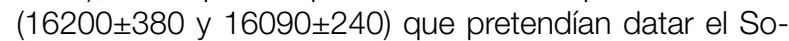
lutrense, pero que corresponderían al Magdaleniense Inferior (EDESO, MUJIKA, 2005: 51). Más hacia el este de esta cavidad se sitúa la de Aitzbitarte IV (Errenteria) con una fecha de 17950 $\pm 100 \mathrm{BP}$; la potencia del nivel y la notable extensión del área excavada en el vestíbulo 
haría necesaria también una serie de dataciones de toda la estratigrafía del yacimiento. Finalmente, en la vertiente norte del Pirineo contamos, entre otras, con las cuevas de Isturitz (Izturitze-Donamartiri), Azkonzilo (Irissarri), Harregi (Altzürükü) -esta última recientemente no ha podido ser satisfactoriamente datada- (ÉBRARD et al., 2013), Brassempouy (Brassempouy) y Harpons (Lespugue).

\subsection{El Magdaleniense Inferior}

A la presencia solutrense en el yacimiento de Praileaitz I se superpone directamente otra correspondiente al Magdaleniense Inferior, que es indudablemente la más singular de toda la secuencia. Su cronología es muy coherente, siendo las fechas obtenidas las siguientes: $15190 \pm 50 \mathrm{BP}-18611-18311 \mathrm{cal} \mathrm{BP}-, 15460 \pm 100 \mathrm{BP}$

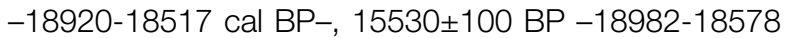
cal BP- y 15810 \pm 110 BP -19397-1827 cal BP-.

A pesar de tratarse de una ocupación importante desde el punto de vista arqueológico, el número de evidencias recuperado es muy reducido, resultado, posiblemente, de una o varias breves estancias, como se intuye por el bajo número de efectivos industriales (MUJIKAALUSTIZA, 2017), así como por los escasos indicios de manipulación antrópica observados entre los restos faunísticos (CASTAÑOS, CASTAÑOS, 2017). Así mismo, las evidencias de carbón recuperadas, principalmente en el único hogar detectado, apuntan en esta línea (RUIZALONSO, ZAPATA, 2017).

La industria ósea es escasa, como se presume en un asentamiento de estas características, en donde no hay restos de astas susceptibles de ser aprovechados para la fabricación de útiles, ni fragmentos con huellas de aserrado. Sí se han hallado, en cambio, dos azagayas de características poco frecuentes, en particular una de base hendida (PEÑALVER, MUJIKA, 2005b).

Dentro del apartado de la industria lítica sucede algo parecido. No hay núcleos, y la desproporción existente entre lo retocado (representa el $28,7 \%$ del total) y lo no retocado invita a pensar que prácticamente la totalidad del instrumental se aportó ya elaborado. En la industria retocada, de carácter laminar, predominan los elementos de dorso (puntas y laminillas), que habría que vincular con la actividad cinegética, mientras que los restantes estarían relacionados con actividades domésticas de distinto tipo (tratamiento de la caza, etc.) (MUJIKA-ALUSTIZA, 2017). Entre estos restos no se observa el número suficiente de instrumentos que pudiera estar directamente implicado en la fabricación de todas las piezas que constituyen el singular lote de colgantes localizados en este nivel y del que se hablará más adelante, por lo que desde el inicio se relegó la hipótesis de que se estuviera ante un posible taller (PEÑALVER, MUJIKA, 2005a). Los estudios de funcionalidad realizados sobre los restos líticos del Magdaleniense Inferior van en esta misma dirección, destacando, con un 60\%, los empleados para el aprovechamiento de recursos animales, tanto en lo que se refiere a su captura (proyectiles), como al aprovechamiento de la carne como recurso alimenticio o a la explotación de las pieles y los huesos como materia prima (CLEMENTE et al., 2017).

En este período, el área central de habitación se sitúa en el vestíbulo, sobre un suelo formado por abundantes clastos de caliza de reducido tamaño, cuya acumulación pudiera tener un origen antrópico, aunque resulte sorprendente el esfuerzo que requeriría esta preparación para estancias temporalmente breves (PEÑALVER, MUJIKA, 2003 y 2005a; PEÑALVER, MUJIKA-ALUSTIZA, 2017a). En esta zona intensamente iluminada se ha localizado un gran bloque calizo de forma propicia para ser utilizado como asiento y tres piedras planas para apoyo de los pies. Justo delante se ha hallado un hogar en cubeta en el que se ha utilizado como combustible madera correspondiente a enebro (Juniperus sp.) (RUIZ-ALONSO, ZAPATA, 2017), conteniendo restos de fauna, así como una azagaya decorada. Parece claro que en torno a este hogar se desarrollaría gran parte de la actividad, localizándose en sus cercanías la mayoría de los restos arqueológicos.

Aunque hasta cierto punto esto es así, la escasez de materiales impide destacar alguna zona concreta de funcionalidad definida por su densidad o por la concentración de determinadas piezas. No obstante, al sur y al oeste del asiento se descubrieron varios fragmentos de ocre (algunos con profundas facetas de abrasión) (PEÑALVER, MUJIKA, 2017a; QUEFELEC et al., 2017); junto a la pared oriental, cerca de la entrada de la cavidad se localizó un conjunto de huesos en conexión anatómica aparentemente preparados para ser procesados en el hogar, situado a tan solo $80 \mathrm{~cm}$. En este mismo espacio se hallaron tres colgantes líticos, formando una agrupación. Otro colgante lítico, roto a la altura de la perforación y al que le falta una parte, se situaba así mismo en la zona de la entrada de la cavidad, al noroeste del hogar.

Por otra parte, en el cubículo situado en la parte trasera del asiento, y muy próximo a él, se hallaron 5 colgantes, casi todos ellos decorados -2 en piedra y 3 en diente-, llamando la atención el que en este pequeño y recogido espacio se produzca esta concentración de piezas de arte mueble de gran relevancia por sus formas: una de ellas se asemeja a una figura femenina, a modo de las venus centroeuropeas, y otra, a un canino atrofiado de ciervo (PEÑALVER, MUJIKA, 2017b; VANHAEREN, D'ERRICO, 2017).

Pero además de la presencia de 9 colgantes en la zona del vestíbulo, otros 20 se extienden por gran parte de la primera sala interior, la mayoría agrupados y en una posición ordenada. De entre todos ellos destaca, por el número de elementos que lo componen (14), por su simetría, por sus decoraciones y por su disposición sobre el terreno en el momento del hallazgo, el conjunto situado en la zona noreste de la primera sala interior. También es de reseñar, en este mismo espacio al que apenas llega la luz natural, la presencia de otra agrupación de tres colgantes en la zona central de la sala, además de otros tres colgantes rotos a la altura de sus respectivas perfo- 
raciones, pero en este caso hallados en la zona oeste de la misma sala.

Entre las características de los colgantes de Praileaitz I destaca la gran homogeneidad de la materia prima empleada para su elaboración, y la mayoría elaborados en lutitas de color negro (D'ERRICO et al., 2017), así como la presencia de decoraciones en la mayor parte de ellos. Estas decoraciones, a base de líneas incisas que ocupan distintas superficies de las piezas, principalmente los lados más estrechos, combinándose de forma diferente, son algo llamativo, al igual que las formas de los cantos seleccionados, en su mayoría alargadas y de color negro. Por otra parte, del análisis tecnológico se desprende que las decoraciones fueron practicadas de forma simultánea, no tratándose por tanto de acumulaciones de marcas realizadas a lo largo de determinado espacio de tiempo (D'ERRICO et al., 2017).

Pero, ¿qué se podría pensar respecto de estos colgantes?. Sabemos en principio que se trata del resultado de la selección de cantos rodados y limonitas de apariencia similar (color gris oscuro/negro), y a veces de formas sugerentes (canino atrofiado, venus, etc.) que posiblemente no fueron recogidos de una sola vez, ni siquiera en el mismo punto, algo fácilmente comprensible si tenemos en cuenta la movilidad de estos grupos. Sin embargo, el hecho de localizarse todos los colgantes en un mismo nivel estratigráfico con varias dataciones que lo confirman, reafirma la sincronía de todos los elementos descubiertos que, ya por sus características, parecían constituir una unidad. Tanto el conjunto de 14 colgantes que parece reflejar una instantánea (PEÑALVER, MUJIKA, 2007-2008), como los otros hallados en esa misma sala y en las distintas zonas del vestíbulo, plasmarían una serie de acontecimientos que hoy no podemos reconstruir, formulando interrogantes a los que tampoco somos capaces de dar respuesta.

La presencia de estos 29 colgantes, que bien pudieron formar parte de distintos collares, o haber estado algunos de ellos (el conjunto de 14) cosidos a una vestimenta (D'ERRICO et al., 2017), o bien haber constituido depósitos votivos, en una cueva "santuario" (recordemos las pinturas existentes al fondo de la cavidad consistentes en líneas y puntos rojos organizadas en series y realizadas con los dedos de cronología paleolítica, aunque no precisa -GARCÍA-DIEZ, OCHOA, 2017-), puede hacernos pensar en algún momento en el acaparamiento de elementos de adorno que se observa en algunos inhumados paleolíticos, y que probablemente portaban en vida como signo de condición, ostentación o por su simbolismo. Es por ello que la asociación de estos colgantes de Praileaitz I con algún individuo concreto, sería plausible.

La caída del gran bloque desprendido de la pared de la entrada, que pudo ser la causa de un abandono precipitado de la cueva, afectó a este conjunto de piezas líticas directamente, partiendo uno de los colgantes en dos, al igual que a la concentración de fauna acumulada cerca del asiento y del hogar, la cual quedó aplastada debajo del bloque en conexión anatómica.

En definitiva, este episodio de habitación efímero de cronología Magdaleniense Inferior ha aportado escasos restos arqueológicos generados por un pequeño grupo humano, no respondiendo a las características de los modelos de cazadero estacional especializado, ni a un alto de caza, ni a un taller. Su singularidad (¿personal, espacial, ritual, simbólica ...?) reside en el conjunto de colgantes, y sobre esta cuestión se podrá profundizar en el futuro en la medida que se incremente el número de situaciones excepcionales equiparables.

El individuo o grupo que ocupó este nivel atribuible al Magdaleniense Inferior habría sido contemporáneo de otros que habitaron en una serie de cuevas próximas. En principio, es muy probable que fuese el yacimiento de Ermittia, situado a tan solo 350 m, con el que Praileaitz I habría estado más estrechamente ligado. Precisamente las ocupaciones más densas de esa cueva son las que están también presentes en Praileaitz I, si bien, la información relativa a Ermittia se ve limitada por la fecha en que se practicó su excavación (1925-1928) (ARANZADI, BARANDIARAN, 1928), así como por las dificultades que presenta su estratigrafía, no siendo posible individualizar los vestigios propios de cada una de las ocupaciones magdalenienses. Este asentamiento estaba especializado en la caza de cabra, animal más consumido así mismo en Praileaitz I, siguiéndole en importancia el ciervo y el sarrio, aportando las fechas de $14900 \pm 165$ y 15420 \pm 145 BP (ESPARZA, MUJIKA, 1999), esta última coincidente con las del hogar y los colgantes de Praileaitz I. Además, la posible relación entre ambas cuevas se refuerza por la presencia de una serie de colgantes sobre piezas dentarias, similares a las descritas en Praileaitz I (PEÑALVER, MUJIKA, 2017a), concretamente uno realizado en Ermittia sobre incisivo de cabra y otro sobre incisivo de caballo, con la misma decoración. Quizá ambas cavidades llegaron a ser complementarias, y la de Praileaitz I, además de su función más específica o peculiar, pudo también servir eventualmente de cobijo o escondrijo durante alguna de las actividades cinegéticas.

Otra cavidad con la que Praileaitz I pudo haber estado relacionada por su proximidad es la de Urtiaga, donde, sobre un Solutrense Final poco característico, existió una ocupación del Magdaleniense Inferior datada en $15530 \pm 70 \mathrm{BP}$ (AMS) y 15620 $\pm 290 \mathrm{BP}$ (conv.) (MUJIKA, 1993; MUJIKA, PEÑALVER, 2012; SUÁREZ-BILBAO, A. et al., 2016), prácticamente contemporánea a la de Praileaitz I. Este nivel, estudiado de forma conjunta con el Solutrense infrayacente, se caracteriza por corresponder a un asentamiento estacional de la época templada del año, en el cual predomina la caza del ciervo $(67,5 \%)$, seguida de la cabra montés (13,6\%).

Así mismo, en el entorno más próximo podríamos señalar el nivel VII de la cueva de Ekain (ALTUNA, MERINO, 1984; MERINO, 1984) una ocupación especializada en la caza de cervatillos (con 5 fechas entre 15510 \pm 270 BP y $15400 \pm 240$ BP); el nivel V de Erralla especializado 
en la caza de cabra montés, datado en $15740 \pm 240$ BP, $16200 \pm 240$ BP y $16270 \pm 240$ BP (ALTUNA et al., 1985), $y$, con cautela, el nivel IV de Amalda, caracterizado por el predominio de la caza de sarrio, y con dos fechas $(16090 \pm 240$ BP y $16200 \pm 380$ BP) obtenidas al tratar de datar el Solutrense (ALTUNA et al., 1990).

Otras ocupaciones de la misma fase cultural se hallarían en la amplia cavidad de Aitzbitarte IV que presenta un denso yacimiento donde se suceden los periodos de habitación sin solución de continuidad, pero sin una delimitación estratigráfica estricta que permita concretar las características particulares de cada uno de ellos. También hay indicios no datados en Bolinkoba (Abadiño), especializado en la caza de cabra (CASTAÑOS, 1983), y en Antoliña, yacimiento en estudio, que ha aportado dos fechas algo más recientes que las de Praileaitz I: $14680 \pm 80$ BP y $14680 \pm 100$ BP (AGUIRRE, 2000; AGIRRE et al. 2000).

\subsection{El Magdaleniense Superior-Final y el Epi- paleolítico}

Al parecer, tras su desocupación durante el Magdaleniense Medio, la cavidad de Praileaitz I se volvió a frecuentar, quizás de forma recurrente, pero siempre de manera fugaz, a lo largo del Magdaleniense SuperiorFinal y del Epipaleolítico, no siendo fácil definir los límites de ambos periodos. En el nivel III, atribuible al Magdaleniense Superior-Final, la cifra de efectivos es baja (94 ejemplares de sílex), entre los que destacan, por su número, los restos brutos de talla, donde hay una elevada proporción de lascas $(68,3 \%)$ frente al de las láminas $(31,7 \%)$, por ejemplo, si lo comparamos con el nivel suprayacente donde las lascas representan el 38\% y las láminas el $62 \%$. Este hecho se explica por la presencia de lascas de retoque y de reavivado halladas en un reducido espacio y a similar cota, y que indicaría la existencia de un breve episodio de talla (acondicionamiento de núcleos, etc.). El número de objetos retocados es muy discreto (14 efectivos -14,6\%-), pero la variabilidad tipológica es amplia, ya que se encuentran representados, aunque con un bajo número, los raspadores, raederas, écaillé, buriles, truncaduras y piezas de dorso (laminillas y puntas). En este aspecto muestra similitud con el nivel II, aunque en el nivel III la proporción de útiles es netamente inferior (MUJIKA-ALUSTIZA, 2017).

Esta ocupación del Magdaleniense Superior-Final tiene cierta entidad únicamente en el vestíbulo y en la zona de tránsito entre este y la primera sala interior, no habiéndose hallado elementos bien estratificados de esta cronología en el resto de los espacios excavados. El único objeto significativo de este periodo cultural es un fino arpón de una hilera de dientes, con paralelos próximos en Ermittia y Urtiaga (MUGICA, 1983), procedente del pasillo de tránsito entre el vestíbulo a la primera sala interior, lugar del que no se tiene datación por la dificultad de seleccionar alguna muestra con garantías. Una datación de C14, obtenida a partir de un fragmento de asta de matanza de reno hallado en el vestíbulo, proporcionó la fecha de 12920 \pm 50 BP (15667-15231 cal BP).

Sobre este asentamiento magdaleniense se desarrollan sucesivas ocupaciones muy breves durante el Epipaleolítico, poco características desde el punto de vista arqueológico, y cuya delimitación estratigráfica es compleja dada la homogeneidad de los sedimentos. Además, la lentitud del proceso de sedimentación, la irregularidad del suelo del depósito y los pequeños buzamientos existentes, también dificultan concretar la cronología de alguno de los objetos más notorios del depósito. A pesar de ello sabemos que existen al menos dos o tres breves momentos en los que distintos grupos humanos ocuparon la cavidad al final del Pleistoceno y al inicio del Holoceno.

En este período el número de evidencias líticas (96) es similar al de la ocupación magdaleniense, pero la proporción de los objetos retocados es notablemente mayor, ya que se incrementa al $24 \%$. La diferencia respecto del nivel magdaleniense radicaría en que en estas fechas no hubo un episodio de talla/retoque tan destacado, aunque existen algunas evidencias muy claras de esta actividad (3 núcleos, un reavivado de núcleo -cuña- y una lámina cresta). Por otra parte, el utillaje es similar al del nivel subyacente, destacando 9 elementos de dorso (3 puntas y 6 laminillas de dorso), además de 2 buriles, 2 raspadores, 4 piezas denticuladas, etc. (MUJIKA-ALUSTIZA, 2017).

La industria ósea, como es habitual en estos períodos, es pobre; cuenta con un posible anzuelo, aunque indudablemente destaca, entre los restos recuperados, un singular bastón perforado con forma o silueta que sugiere la cabeza de un ave (¿buitre?) con un gran orificio de suspensión; este orificio es excesivamente grande para esta finalidad, e ineficaz para los hipotéticos usos de estos objetos. Inicialmente, esta pieza tal vez pudiera haber contado con varias perforaciones y ser posteriormente reutilizada tras algunas modificaciones hoy irreconocibles. Desde el punto de vista tipológico parece un objeto magdaleniense, a pesar de que también se conocen bastones o candiles perforados similares en cronologías más modernas (Logalan, Herriko Barra, etc.) (EDESO, MUJIKA, 2005). Además, en favor de esta cronología está la semejanza de su silueta con la del extremo apuntado del "cetro" de La Vache, objeto que a su vez tiene un paralelo tipológico próximo en un ejemplar de Santa Catalina (datado en torno a $12345 \pm 85$ BP y $12425 \pm 90$ BP -BERGANZA et al., 2014-) y otro en Isturitz (MUJIKA, 1993).

La fecha aportada por el bastón de Praileaitz I es 10740 \pm 50 BP (12738-12594 cal BP), es decir Aziliense, y prácticamente coincide con la aportada por una Littorina obtussata (10560 \pm 50 BP -12621-12114 cal BP-) recogida en la zona de tránsito del vestíbulo a la primera sala interior (RIGAUD et al., 2017). Otra concha, Littorina saxatilis, procedente de un contexto de más de dos centenares de piezas de pequeño tamaño, sin perforar, fue datada en 9320 \pm 40 BP (10571-10252 cal BP). Estas 
conchas tal vez fueron fueran recogidas con la intención de servir para la elaboración de colgantes, aunque fueron abandonadas en el yacimiento sin ser modificadas, localizándose de forma concentrada en la parte norte y centro de la primera sala interior y en la zona sur del pasillo formado entre el vestíbulo y la primera sala (RIGAUD et al., 2017).

Los dos hogares detectados sobre este nivel de caracoles marinos no cuentan con restos materiales asociados a ellos, ni tampoco existen evidencias de su utilización cotidiana; por otra parte, uno de ellos, de $2 \mathrm{~m}$ de diámetro, se hallaba ubicado en una zona compleja por ser un lugar de tránsito. Ambas estructuras han sido datadas en: $8840 \pm 40 \mathrm{BP}(10157-9741 \mathrm{cal} \mathrm{BP}), 8840 \pm 45$ BP (10157-9734 cal BP) y 9010 \pm 50 BP. (10250-10122 cal BP), documentando un claro episodio de ocupación durante el Epipaleolítico. Las fechas obtenidas a partir de restos de carbones hallados en la segunda sala interior (8800 \pm 35 BP -10120-1064 cal BP-, 8925 \pm 40 BP $-10201-10111$ cal BP-, 9200 \pm 40 BP $-10491-10455$ cal BP- y $9225 \pm 40$ BP $-10505-10260$ cal BP- ) en la que los restos industriales son muy escasos, son coincidentes con las de los dos hogares, por lo que, probablemente, los carbones debieron llegar a esa zona interior por causas difíciles de concretar, tal vez arrastradas por el agua. Sin embargo, no existe, aparte de los hogares, ningún elemento objetivo que permita concretar los restos materiales de esta ocupación en el vestíbulo. Tras esta ocupación de finales del Epipaleolítico Antiguo, o ya ligeramente más reciente, la presencia humana prehistórica no dejó rastro detectable alguno en la cavidad.

Durante el periodo en que se produjeron estas breves estancias del Magdaleniense Superior-Final y del Epipaleolítico Antiguo, esta cavidad, y tal vez a causa del obstáculo que el gran bloque desprendido con anterioridad en la entrada, y que la tapaba casi en su totalidad, no constituiría más que un asentamiento muy provisional para desarrollar actividades depredadoras y recolectoras.

A lo largo de ambos períodos (Magdaleniense Superior-Final y Epipaleolítico) la fauna está representada en el vestíbulo de Praileaitz I de forma destacada por la cabra, seguida a considerable distancia por el ciervo y el sarrio, al igual que sucedía durante el Magdaleniense Inferior (CASTAÑOS, CASTAÑOS, 2017). Así mismo, dentro de los niveles correspondientes al Epipaleolítico, se localizan diversos restos de peces pertenecientes a salmónidos del género Salmo y que por el contexto de ubicación pudieran ser atribuidos a la trucha de río (Salmo trutta fario), lo que de ser así apuntaría a un origen local, concretamente a las aguas dulces colindantes con el yacimiento (ROSELLÓ, MORALES, 2017).

El número de yacimientos conocidos en este entorno cárstico durante el Magdaleniense Superior-Final y el Epipaleolítico aumenta de forma considerable al haberse reducido el territorio explotable existente entre el litoral y los primeros cordales de montaña, como consecuencia de la subida de las temperaturas y la consiguiente ele- vación del nivel marino, lo que propició que importantes extensiones de territorio quedasen sumergidas bajo el agua. Es por ello que a partir del Magdaleniense Final, cavidades como Urtiaga o Ermittia, entre otras, adquirirían un carácter costero.

En este contexto, destaca la cavidad de Ermittia que cuenta con una ocupación del Magdaleniense SuperiorFinal, fechada en $12525 \pm 115 \mathrm{BP}$, a la que se superpone un característico Aziliense, que no pudo ser datado de forma satisfactoria (ESPARZA, MUJIKA, 1999). En esta fecha continúa siendo este lugar un cazadero de cabras (57\%), si bien aumenta de forma importante el ciervo, que alcanza el 24\%, lo que quizás fuese debido a la mejora climática. Los indicios de marisqueo son muy discretos.

En el mismo entorno se encuentran las cuevas de Langatxo e Iruroin, situadas a 350 y 750 m respectivamente, con evidencias del Magdaleniense Final (ZUMALABE 1994 y 1999). Es muy probable que estos yacimientos hubieran estado interrelacionados en esas fechas, así como los de Aizkoltxo (MURELAGA et al., 2008) y Agarre, que se localizan curso arriba, a unos $4 \mathrm{~km}$ junto al río Deba el primero, y junto a un afluente (Kilimon) el segundo. El primero de ellos presenta un rico nivel atribuible al Magdaleniense Final, con abundante industria lítica y ósea (buriles, dorsos, arpones magdalenienses y alguno de transición al Aziliense, azagayas, etc.) datado en $12380 \pm 60 \mathrm{BP}$, y otro nivel Aziliense datado en 9980 \pm 65 BP. El segundo cuenta, bajo una típica industria Aziliense, con evidencias características del Magdaleniense Final (azagayas de doble bisel reutilizadas como cincel, una base de arpón, etc.) (MÚGICA, 1983, MUJIKA, 1993).

Otra cavidad a tener en cuenta en este entorno geográfico es la de Urtiaga, situada, como se ha citado anteriormente, a unos $4 \mathrm{~km}$ al noreste de Praileaitz I. Posee unas densas ocupaciones azilienses (el nivel $C$ fue datado en $8700 \pm 100 \mathrm{BP}$ ) y magdalenienses, siendo a partir de la fase final cuando se incrementa de forma notable el marisqueo (principalmente de lapas). La parte superior del nivel $D$, en opinión de J. $M^{a}$ Merino (hipótesis que compartimos por el descenso de efectivos de la industria ósea), sería probablemente Aziliense, con una fecha de $10280 \pm 190$ BP. Durante el Magdaleniense este lugar fue utilizado como cazadero principalmente de ciervo (47\%), junto a otras especies como el corzo, el sarrio y la cabra, con aproximadamente un $12 \%$ de representatividad cada una de ellas. Durante el Aziliense aumenta la presencia de ciervo $(63,5 \%)$ y corzo $(15 \%)$, y en especial de jabalí (7\%), mientras que el sarrio y la cabra ven reducida su presencia al 7\% (ALTUNA, 1990).

En la cueva de Ekain (ALTUNA, MERINO, 1984) se constata también la presencia de ambos periodos, siendo datado el Magdaleniense Final (nivel VI) en

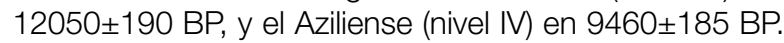
En este asentamiento estacional, en una primera fase se dedican mayoritariamente a practicar la caza de la cabra (71,1\%), actividad que se reduce drásticamente durante 
el Aziliense (10,6\%), mientras que el ciervo, de estar representado por el 20,9\% de los restos en el primer período, pasa a alcanzar el 85,2\%. No hay indicios de actividad mariscadora en el Magdaleniense Final, y es escasa en el Aziliense, probablemente debido a que esta tarea no se realizaría desde Ekain, sino que los pocos restos hallados serían aportados por grupos procedentes del litoral que acudirían a la cavidad llevando consigo algunos enseres y provisiones.

Santa Catalina, en la cuenca del Lea, es otro de los yacimientos próximos y contemporáneos con Praileaitz I. En él, dentro de los niveles III-I, se desarrollan interesantes ocupaciones del Magdaleniense Superior-Final y Aziliense, que se situarían entre la fecha más antigua de $12425 \pm 90$ BP y la más reciente de $9180 \pm 110$ BP. Presenta una serie de características singulares como la riqueza taxonómica y la abundancia de restos de malacofauna, de ictiofauna (destacan el salmón, el bacalao, los pleuronéctidos, etc.) y de avifauna. Entre la macrofauna, en la que predomina el ciervo, sobresale la presencia de reno hasta la etapa más reciente, ya en el Holoceno. Indudablemente esta cueva representa una nueva modalidad de asentamiento que enriquece nuestra visión sobre los modos de vida de estas poblaciones prehistóricas (BERGANZA et al. , 2012; BERGANZA, ARRIBAS, 2014).

Otro enclave, algo más alejado, pero que conserva ocupaciones de la misma cronología, es Santimamiñe, excavado en su mayor parte hace casi un siglo, pero del que los trabajos más recientes permiten poner en valor (LÓPEZ QUINTANA et al., 2011). La existencia de un Magdaleniense Final en la zona interior excavada no ha podido ser certificada de forma satisfactoria mediante datación, pero existen al menos dos fechas para el Aziliense $(10100 \pm 60$ BP y $10060 \pm 60$ BP).

En el entorno al que nos estamos refiriendo existe otra serie de yacimientos que únicamente enumeraremos, ya que la información disponible es limitada por tratarse de excavaciones antiguas y sin dataciones (Lumentxa, Abittaga, Silibranka), o por ser yacimientos con niveles parcialmente conservados o alterados (Laminak

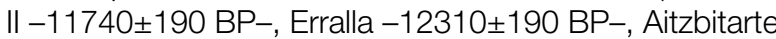
IV) (ALTUNA et al., 1985; GONZÁLEZ SAINZ, 1989; MUJIKA-ALUSTIZA, 1983 y 1993; BERGANZA, ARRIBAS, 1994).

En resumen, la cueva de Praileaitz I presenta una notable secuencia de ocupación enmarcada en el Tardiglaciar y en los inicios del Holoceno conservando restos estratificados que comienzan en el Solutrense y sobre los que se superponen vestigios de breves episodios de habitación durante el Magdaleniense Inferior, el Magdaleniense Superior-Final y el Epipaleolítico avanzado. En todas estas fases se trata de un asentamiento que corresponde a reducidos grupos humanos que, probablemente, estuvieron estrechamente vinculados a una red de ocupaciones más o menos próximas. Esta red de asentamientos constituiría complejos circuitos diseñados para una racional gestión del medio, en el que serían básicos los conocimientos sobre la movilidad estacional de las distintas especies animales, los ciclos de desarrollo de la vegetación, las fuentes de materia prima, etc., produciéndose paralelamente sólidas relaciones intergrupales.

Pero la peculiaridad de Praileaitz I en este importante contexto reside en la ocupación del Magdaleniense Inferior, a pesar de que quede sin resolver de manera plenamente satisfactoria su función en este momento y el porqué de la presencia de un cuantioso número de colgantes decorados, de singulares características, fabricados sobre soportes pocos frecuentes, así como de otros elementos asociados a ellos. No obstante, esta serie de materiales no parecen guardar una relación directa con actividades de tipo cotidiano y sí, quizá, con otras de tipo cultual.

\section{7.- BIBLIOGRAFÍA}

\section{AGUIRRE RUIZ DE GOPEGUI, M.}

2000 El yacimiento paleolítico de Antoliñako Koba (Gautegiz-Arteaga, Bizkaia): secuencia estratigráfica y dinámica industrial. Avance de las campañas excavación 1995-2000. Illunzar 4, 38-81.

AGUIRRE RUIZ DE GOPEGUI, M., LÓPEZ QUINTANA, J.C., SÁENZ DE BURUAGA, A.

2000 Medio ambiente, industrias y poblamiento prehistórico en Urdaibai. Illunzar 98/00, 4, 13-38.

\section{AGUIRRE RUIZ DE GOPEGUI, M.: GONZÁLEZ SAINZ, C.}

2011 Placa con grabado figurativo del Gravetiense de Antoliñako koba (Gautegiz-Arteaga, Bizkaia). Implicaciones en la caracterización de las primeras etapas de la actividad gráfica en la región Cantábrica. Kobie 30, 43-62.

ALTUNA, J.

1990 La caza de herbívoros durante el Paleolítico y Mesolítico del País Vasco. Munibe 42, 229-240.

ALTUNA, J; MERINO, J. M. (DIR)

1984 El yacimiento prehistórico de la Cueva de Ekain (Deba, Guipúzcoa). Sociedad de Estudios Vascos. San Sebastián

ALTUNA, J.; BALDEÓN, A.; MARIEZKURRENA, K.

1985 Cazadores magdalenienses en la Cueva de Erralla (Cestona, País Vasco). Munibe 37

ALTUNA, J.; BALDEÓN, A.; MARIEZKURRENA, K. et al.

1990 La Cueva de Amalda (Zestoa, País Vasco). Ocupaciones paleoliticas y postpaleolíticas. Sociedad de Estudios Vascos. Donostia. 
ARANBURU, A., ARRIOLABENGOA, M., IRIARTE, E., VADILLO, P., ARESO, P., URIZ, A.

2017 Estudio geológico de la cavidad de Praileaitz I (Deba,Gipuzkoa), en PEÑALVER, X., SAN JOSE, S., MUJIKA-ALUSTIZA, J.A. La cueva de Praileaitz I (Deba, Gipuzkoa, Euskal Herria). Intervención arqueológica 2000 - 2009. Munibe Monographs. Anthropology and Archaeology Series 1, 135-169.

ARANZADI, T.; BARANDIARAN, J.M.

1928 Exploraciones prehistóricas en Guipúzcoa los años 1924 a 1927. Cavernas de Ermittia (Sasiola), Arbil (Lastur) y Olatzaspi (Asteasu), dolmen de Basagain (Murumendi) y caverna de Iruritxo (Nergara), en: BARANDIARAN, J.M. Obras completas X, 169-261. Bilbo.

BERGANZA, E.; ARRIBAS, J. L.; CASTAÑOS, P.; ELORZA, M.; GONZÁLEZ URQUIJO, J. E.; IBAÑEZ, J. I.; IRIARTE, Mª J.; MORALES, A.; PEMÁN, E. ROSALES, T.; ROSELLÓ, E.; RUIZ IDARRAGA, R.; URIZ, A.; UZQUIANO, P.; VÁSQUEZ, V.; ZAPATA, L.

2012 La transición tardiglaciar en la costa oriental de Bizkaia: el yacimiento de Santa Catalina. Resultados preliminares, en ARIAS, P.; CORCHÓN, Ma S.; MENDÉNDEZ, M.; RODRíGUEZ, J. A. (Eds.). El Paleolítico Superior Cantábrico. Actas de la Primera Mesa Redonda. San Román de Candamo (Asturias), 26-28 abril de 2007, 97-113. Universidad de Cantabria. Santander.

BERGANZA-GOCH, E.; ARRIBAS PASTOR, J. L.

1994 Excavación, cronología, análisis de las industrias lítica y ósea de Lamiñak II. Kobie XXI, 7-83.

BERGANZA-GOCHI, E.; ARRIBAS PASTOR, J. L. et al.

2014 La Cueva de Santa Catalina (Lekeitio): La intervención arqueológica. Resto vegetales, animales y humanos. Kobie. Bizkaiko Arkeologi Indusketak 4.

BERGANZA, E.; RUIZ IDARRAGA, R.

2014 Candil de ciervo decorado magdaleniense del yacimiento de Santa Catalina (Bizkaia, España). Tecnología y funcionalidad. Munibe 65, 25-36.

CASTAÑOS, P., CASTAÑOS, J.

2017 Estudio de la fauna de macromamíferos del yacimiento de Praileaitz I (Deba, Gipuzkoa), en PEÑALVER, X., SAN JOSE, S., MUJIKA-ALUSTIZA, J.A. La cueva de Praileaitz I (Deba, Gipuzkoa, Euskal Herria). Intervención arqueológica 2000 - 2009. Munibe Monographs. Anthropology and Archaeology Series 1, 221-265.

CASTAÑOS UGARTE, P. M M $^{2}$

1983 Estudio de los macromamiferos del yacimiento prehistorico de Bolinkoba (Abadiano-Vizcaya). Kobie XIII, 261 298.

CLEMENTE, I., GARCÍA, V., VILA, A.

2017 Análisis funcional de los restos líticos tallados de la cueva de Praileaitz I (Deba, Gipuzkoa), en PEÑALVER, X., SAN JOSE, S., MUJIKA-ALUSTIZA, J.A. La cueva de Praileaitz I (Deba, Gipuzkoa, Euskal Herria). Intervención arqueológica 2000 - 2009. Munibe Monographs. Anthropology and Archaeology Series 1, 399-409.
CORCHÓN RODRÍGUEZ, Ma S.

1993 El magdaleniense con triángulos de Las Caldas (Asturias, España). Nuevos datos para la definición del Magdaleniense inferior Cantábrico. Zephyrus 46, 77-94.

D'ERRICO, F., VANHAEREN, M., QUEFFELEC, A.

2017 Les galets perforés de Praileaitz I (Deba, Gipuzkoa), en PEÑALVER, X., SAN JOSE, S., MUJIKA-ALUSTIZA, J.A. La cueva de Praileaitz I (Deba, Gipuzkoa, Euskal Herria). Intervención arqueológica 2000 - 2009. Munibe Monographs. Anthropology and Archaeology Series 1, 453484.

EBRARD, D.; SÁENZ DE BURUAGA, A.; MUJIKA-ALUSTIZA, J. A., NÉVÉAL, R.

2013 La grotte de Harregi à Aussurucq (Pyrénées-Atlantiques). 50 ans d'archéologie en Soule. Hommage à Pierre Boucher (1909-1997), Ikerzaleak 7, 59-73.

EDESO, J. M.; MUJIKA, J. A.

2005 El entorno de Zarautz durante el Cuaternario. Evolución paisajistica, ambiental y humana. Museo de Arte e Historia de Zarautz. Zarautz.

EDESO, J. M.; MUJIKA-ALUSTIZA, J. A

2006 La cueva de Aizkoltxo. Arkeoikuska 2005, 187-190.

EDESO-FITO, J.M., MUJIKA-ALUSTIZA, J.A.

2017 Estudio de patrones espaciales y de reconstrucción paisajística mediante el uso de sistemas de información geográfica (Praileaitz I, Deba, Gipuzkoa), en PEÑALVER, X., SAN JOSE, S., MUJIKA-ALUSTIZA, J.A. La cueva de Praileaitz I (Deba, Gipuzkoa, Euskal Herria). Intervención arqueológica 2000 - 2009. Munibe Monographs. Anthropology and Archaeology Series 1, 171-191.

ESPARZA, X.; MUJIKA, J. A.

1999 Reflexiones en torno a la estratigrafía de Ermittia (Deva, Guipúzcoa). Congreso Nacional de Arqueología XXIV (Cartagena, 1997), 61-69. Cartagena.

GARCÍA-DIEZ, M., OCHOA, B.

2017 Arte rupestre en la cueva de Praileaitz I (Deba, Gipuzkoa),

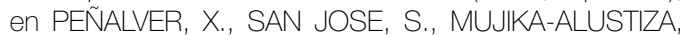
J.A. La cueva de Praileaitz I (Deba, Gipuzkoa, Euskal Herria). Intervención arqueológica 2000 - 2009. Munibe Monographs. Anthropology and Archaeology Series 1, 505-513.

GARCIA-IBAIBARRIAGA, N., BAILON, S, ROFES, J, ORDIALES, A, SUÁREZ-BILBAO, A., MURELAGA, $X$

2017 Estudio de los microvertebrados del yacimiento de Praileaitz I (Deba, Gipuzkoa), en PEÑALVER, X., SAN JOSE, S., MUJIKA-ALUSTIZA, J.A. La cueva de Praileaitz I (Deba, Gipuzkoa, Euskal Herria). Intervención arqueológica 2000 - 2009. Munibe Monographs. Anthropology and Archaeology Series 1, 267-285.

GONZÁLEZ SAINZ, C.

1989 El Magdaleniense Superior-Final de la región cantábrica. Tantin. Universidad de Cantabria. Santander. 
IRIARTE-CHIAPUSSO, Ma J

2017 Praileaitz I (Deba, Gipuzkoa): el estudio paleopalinológico, en PEÑALVER, X., SAN JOSE, S., MUJIKA-ALUSTIZA, J.A. La cueva de Praileaitz I (Deba, Gipuzkoa, Euskal Herria). Intervención arqueológica 2000 - 2009. Munibe Monographs. Anthropology and Archaeology Series 1, 193-199.

LÓPEZ QUINTANA, J. C.; et al.

2011 La cueva de Santimamiñe: revisión y actualización (20042006). Kobie, Bizkaiko Arkeologi Indusketak. Excavaciones Arqueológicas en Bizkaia 1.

MERINO, J. Ma

1984 Industria lítica del yacimiento Ekain, en ALTUNA, J.; MERI$\mathrm{NO}, \mathrm{J} . \mathrm{M}^{a}$, El yacimiento prehistórico de la cueva de Ekain (Deba, Guipúzcoa), 65-175. Eusko-Ikaskuntza. Donostia.

\section{MORENO-GARCÍA, M.}

2017 Estudio de la avifauna de la cueva de Praileaitz I (Deba, Gipuzkoa), en PEÑALVER, X., SAN JOSE, S., MUJIKAALUSTIZA, J.A. La cueva de Praileaitz I (Deba, Gipuzkoa, Euskal Herria). Intervención arqueológica 2000 - 2009. Munibe Monographs. Anthropology and Archaeology Series 1, 287-321.

MÚGICA, J. A.

1983 La industria de hueso en la Prehistoria de Guipúzcoa. Munibe 35, 451-631.

MUJIKA, J. A.

1993 La industria ósea del Paleolítico Superior y Epipaleolítico del Pirineo Occidental. Universidad de Deusto, Bilbao.

MUJIKA-ALUSTIZA, J.A

2017 Las industrias líticas y óseas de Praileaitz I (Deba, Gipuzkoa), en PEÑALVER, X., SAN JOSE, S., MUJIKAALUSTIZA, J.A. La cueva de Praileaitz I (Deba, Gipuzkoa, Euskal Herria). Intervención arqueológica 2000 - 2009. Munibe Monographs. Anthropology and Archaeology Series 1, 353-390.

MUJIKA ALUSTIZA, J. A.; PEÑALVER IRIBARREN, X.

2012 La ocupación de la cuenca del Deba (Gipuzkoa) durante el Magdaleniense Inferior, en ARIAS, P.; CORCHÓN, Mª S.; MENDÉNDEZ, M.; RODRÍGUEZ, J. A. (Eds.). El Paleolítico Superior Cantábrico. Actas de la Primera Mesa Redonda (2007). San Román de Candamo (Asturias), 97 113. Universidad de Cantabria. Santander.

MURELAGA, X.; MUJIKA-ALUSTIZA, J. A.; BAILON, S.; CASTAÑOS, P., SAEZ DE LAFUENTE, $X$.

2008 La fauna de vertebrados del yacimiento Holoceno (Aziliense) de Aizkoltxo (Mendaro, Gipuzkoa). Geogaceta 45, 71-74.

QUEFELEC, A.; D'ERRICO, F.; VANHAEREN, M.

2017a Analyse des blocs de matière colorante de Praileaitz I (Deba, Gipuzkoa), en PEÑALVER, X., SAN JOSE, S., MUJIKA-ALUSTIZA, J.A. La cueva de Praileaitz I (Deba,
Gipuzkoa, Euskal Herria). Intervención arqueológica 2000 - 2009. Munibe Monographs. Anthropology and Archaeology Series 1, 493-503.

PEÑALVER, $X$.

2013 Praileaitz I leizea. Cueva de Praileaitz. Arkeoikuska 2012, 275-277.

2014 Praileaitz I haitzuloa. La cueva de Praileaitzl (Deba, Gipuzkoa). Gipuzkoako Foru Aldundia. Donostia. (Arkeologia; 4).

PEÑALVER, X.; MUJIKA, J. A.

2003 Suelo de ocupación magdaleniense en la cueva de Praile Aitz I (Deba, Gipuzkoa): evidencias de arte mobiliar. Veleia 20, 157-181.

2005a Praile Aitz I (Deba, Gipuzkoa): evidencias arqueológicas y organización espacial en un suelo Magdaleniense. Promontoria Monográfica 02, O Paleolítico. Actas do IV Congresso de Arqueología Peninsular (Faro, 2004). 143-157. Universidade do Algarve. Faro.

2005b La cuestión de las azagayas de base hendida magdalenienses en la Cornisa Cantábrica. Veleia 22, 9-21.

2007-2008 ¿Actividad cotidiana o práctica ritual?. Agrupación de 14 colgantes líticos del Magdaleniense Inferior en la cueva de Praileaitz I (Deba, Gipuzkoa). Veleia 24-25, 209-228.

PEÑALVER, X., MUJIKA-ALUSTIZA, J.A.

2017a La utilización del espacio en la ocupación de la cueva de Praileaitz I (Deba, Gipuzkoa), en PEÑALVER, X., SAN JOSE, S., MUJIIKA-ALUSTIZA, J.A. La cueva de Praileaitz I (Deba, Gipuzkoa, Euskal Herria). Intervención arqueológica 2000 - 2009. Munibe Monographs. Anthropology and Archaeology Series 1, 121-133.

2017b Los colgantes de la cueva de Praileaitz I (Deba, Gipuzkoa) y sus características formales, en PEÑALVER, X., SAN JOSE, S., MUJIKA-ALUSTIZA, J.A. La cueva de Praileaitz I (Deba, Gipuzkoa, Euskal Herria). Intervención arqueológica 2000 - 2009. Munibe Monographs. Anthropology and Archaeology Series 1, 411-451.

PEÑALVER, X., SAN JOSE, S., MUJIKA-ALUSTIZA,J.A.

2006 Praileaitz I haitzuloko (Deba) Madeleine aldiko zintzilikarioak. Los colgantes magdalenienses de la cueva de Praileaitz I (Deba). Gipuzkoako Foru Aldundia. Donostia. (Bertan; 22).

2017 El yacimiento arqueológico de Praileaitz I (Deba, Gipuzkoa, Euskal Herria). Metodología de la excavación, estratigrafía, estructuras y dataciones arqueológicas, en PEÑALVER, X., SAN JOSE, S., MUJIKA-ALUSTIZA, J.A. La cueva de Praileaitz I (Deba, Gipuzkoa, Euskal Herria). Intervención arqueológica 2000 - 2009. Munibe Monographs. Anthropology and Archaeology Series 1, 33-120.

RIGAUD, S.; D'ERRICO, F.; VANHAEREN, M.

2017 Les coquillages marins de Praileaitz I (Deba, Gipuzkoa), en PEÑALVER, X., SAN JOSE, S., MUJIKA-ALUSTIZA, J.A. La cueva de Praileaitz I (Deba, Gipuzkoa, Euskal Herria). Intervención arqueológica 2000 - 2009. Munibe Monographs. Anthropology and Archaeology Series 1, 333-351. 
RÍOS, J.; GARATE, D.; GÓMEZ, A.; ARCEREDILLO, D.; IRIARTE, E.; GARCÍA, A.; GARRIDO, D.; S. PEDRO, Z.

2013 El final del Solutrense en el oriente cantábrico a través de las ocupaciones de la cueva de Arlanpe (Lemoa, Vizcaya). Zephyrus LXXII, 15-38.

ROSELLO IZQUIERDO, A., MORALES MUÑIZ, A.

2017 Los peces de la cueva de Praileaitz I (Deba, Gipuzkoa), en PEÑALVER, X., SAN JOSE, S., MUJIKA-ALUSTIZA, J.A. La cueva de Praileaitz I (Deba, Gipuzkoa, Euskal Herria). Intervención arqueológica 2000 - 2009. Munibe Monographs. Anthropology and Archaeology Series 1, 327-331.

RUIZ-ALONSO, M.; ZAPATA, L.

2017 Estudio de los macrorrestos vegetales de Praileaitz I (Deba, Gipuzkoa), en PEÑALVER, X., SAN JOSE, S., MUJIKA-ALUSTIZA, J.A. La cueva de Praileaitz I (Deba, Gipuzkoa, Euskal Herria). Intervención arqueológica 2000 - 2009. Munibe Monographs. Anthropology and Archaeology Series 1, 201-219.

SAN JOSE, S. PEÑALVER, X., MUJIKA-ALUSTIZA, J.A.

2017 Praileaitz I haitzuloa (Deba, Gipuzkoa, Euskal Herria). Deskribapena. Testuinguru geografikoa eta arkeologikoa, en PEÑALVER, X., SAN JOSE, S., MUJIKA-ALUSTIZA, J.A. La cueva de Praileaitz I (Deba, Gipuzkoa, Euskal Herria). Intervención arqueológica 2000 - 2009. Munibe Monographs. Anthropology and Archaeology Series 1, 13-31.

SUÁREZ-BILBAO, A.; LARRAZ, M.; MUJIKA-ALUSTIZA, J.A.; SUAREZ-HERNANDO, O.; MURELAGA, $X$.

2016 Gasterópodos continentales del Pleistoceno superiorHoloceno basal de Urtiaga (Deba, Gipuzkoa). Geogaceta 60, 83-86.
TARRIÑO VINAGRE, A.

2017 Procedencia de los sílex recuperados en Praileaitz I (Deba Gipuzkoa), en PEÑALVER, X., SAN JOSE, S., MUJIKAALUSTIZA, J.A. La cueva de Praileaitz I (Deba, Gipuzkoa, Euskal Herria). Intervención arqueológica 2000 - 2009. Munibe Monographs. Anthropology and Archaeology Series 1, 391-398.

URIBARRI, E.

1988 Cueva de Praile Aitz II (Deba, Guipúzcoa). I campaña. Arkeoikuska 88, 103-105.

1989 Cueva de Praile Aitz II (Deba, Guipúzcoa). II campaña. Arkeoikuska 89, 164-16.

VANHAEREN, M.; D'ERRICO, F.;

2017 Les incisives de bouquetin perforees. Analyse archeozoologique et technologique, en PEÑALVER, X., SAN JOSE, S., MUJIIKA-ALUSTIZA, J.A. La cueva de Praileaitz I (Deba, Gipuzkoa, Euskal Herria). Intervención arqueológica 2000 - 2009. Munibe Monographs. Anthropology and Archaeology Series 1, 485-492.

ZUMALABE, FR.

1994 Cueva de Langatxo (Mutriku). IV campaña. Arkeoikuska 93, 168-172.

1999 La cueva de Iruroin (Mutriku). Arkeoikuska 99, 98-101. 
